Supporting Information

\title{
Xeno Free Bioreactor Culture of Human Mesenchymal Stromal Cells on Chemically Defined Microcarriers
}

John D. Krutty'; Kevin Koesser2, Stephen Schwartz²; Junsu Yun'; William L. Murphy ${ }^{1,2,3}$;

and Padma Gopalan $n^{1,2,4 *}$

${ }^{1}$ Department of Materials Science and Engineering, University of Wisconsin-Madison

${ }^{2}$ Department of Biomedical Engineering, University of Wisconsin-Madison

${ }^{3}$ Department of Orthopedics and Rehabilitation, University of Wisconsin-Madison

${ }^{4}$ Department of Chemistry, University of Wisconsin-Madison

1500 University Avenue, Madison, WI 53706 
Number of Figures: 5

Number of Pages: 6 
a

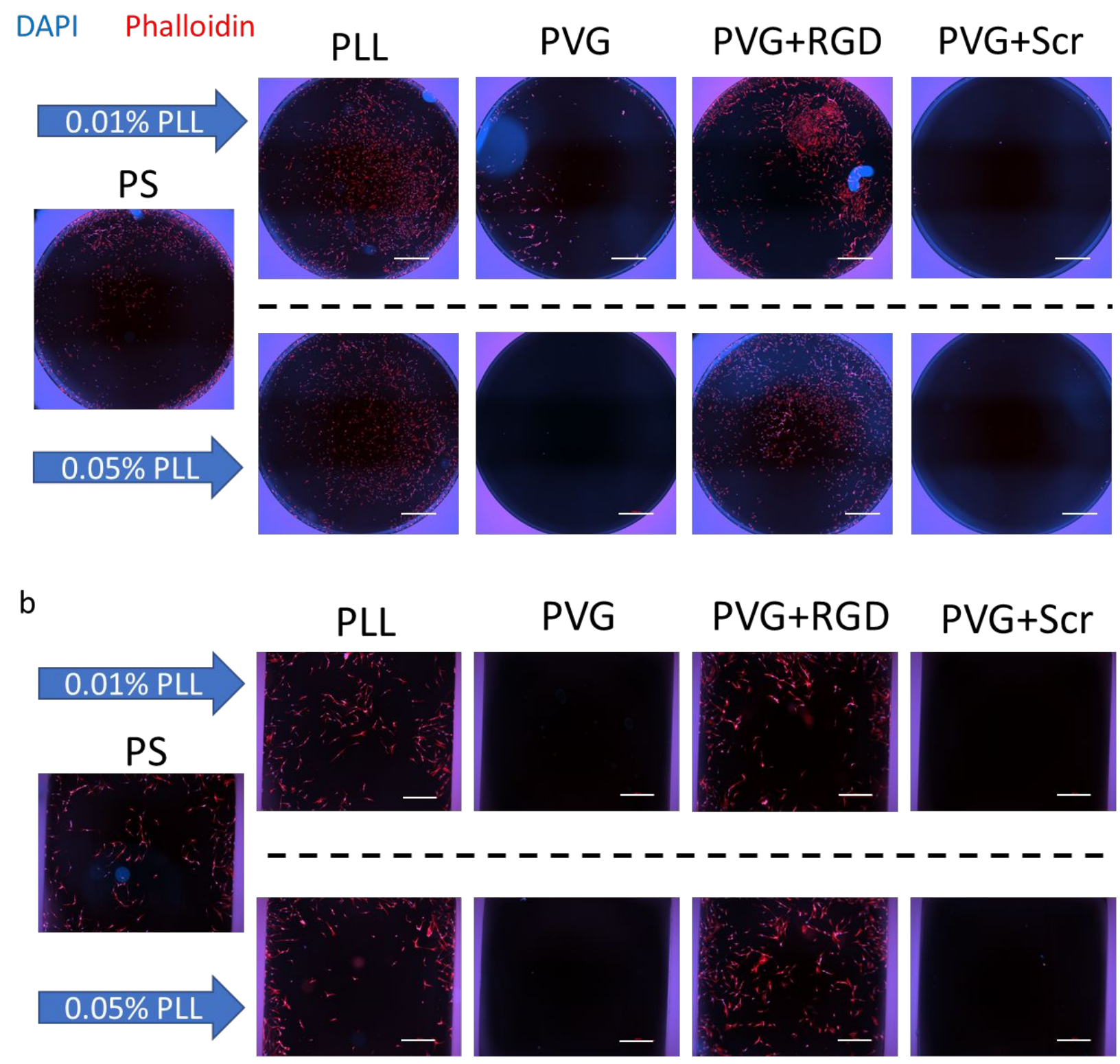

Supplemental Figure S1. Increased PLL content improves the application of PVG to TCPS surfaces. Representative micrographs of hMSC grown on PVG-coated a) 96-well and b)384-well polystyrene plates. a) scale bar $=1000 \mu \mathrm{m}$. b) scale bar $=500 \mu \mathrm{m}$ 


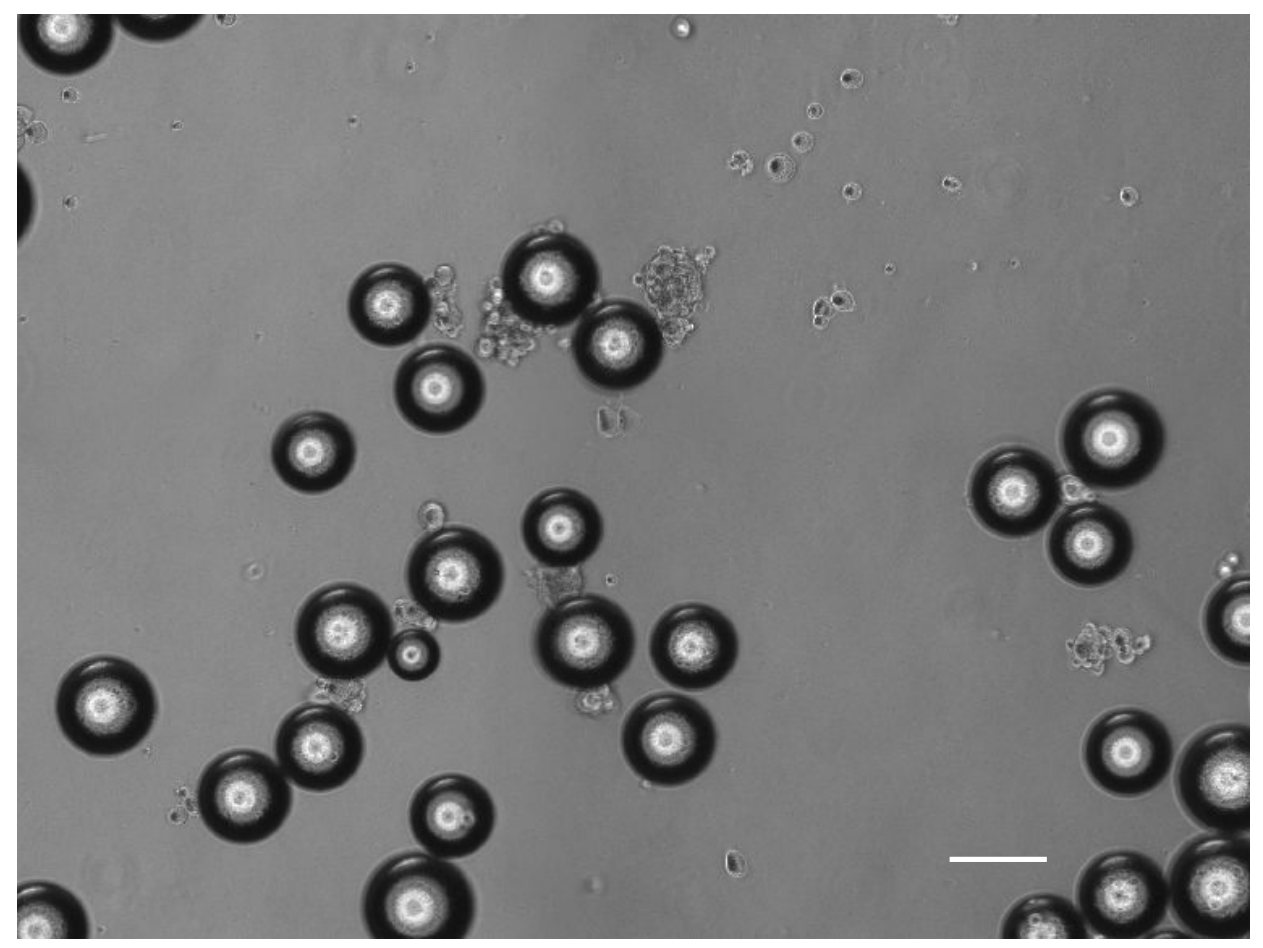

Supplemental Figure S2. hMSC do not adhere to bare PS microcarriers in bioreactor culture in xeno-free media. Phase contrast images of hMSC seeded onto PS microcarriers for $12 \mathrm{~h}$ under static conditions, and stirred at 60rpm for $12 \mathrm{~h}$. Samples removed show many unadhered cells, cells that have formed aggregates, and cells that are loosely attached to microcarriers. Scale bar $=$ $200 \mu \mathrm{m}$. 


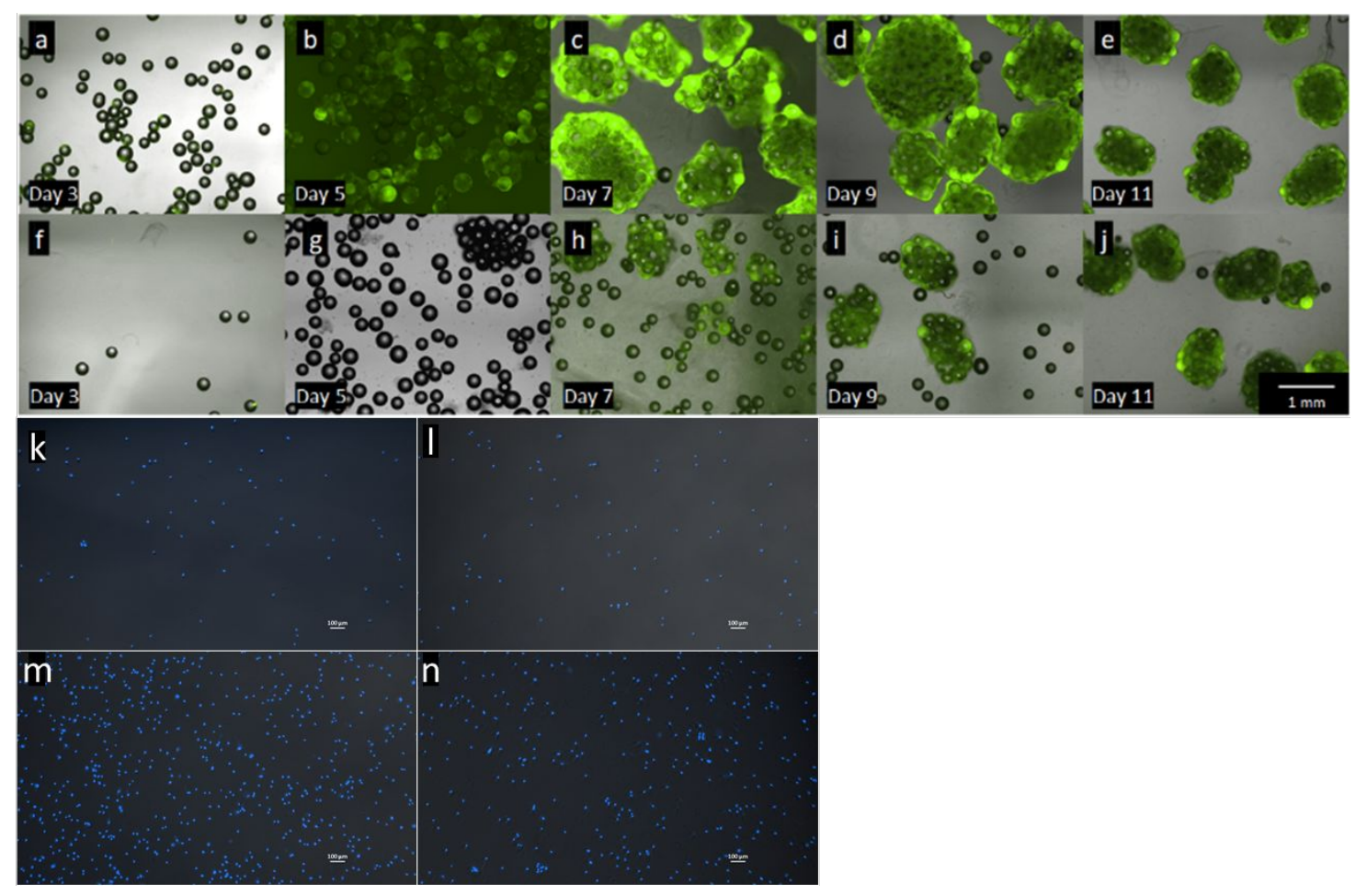

Supplemental Figure S3. 11 day bioreactor culture of hMSC is possible in both in xeno-free (a-e) and serum-containing media (f-j). Microcarriers and cells begin to form large aggregates between day 5 and day 7 . These aggregates continue to get larger out to day 11 . Large aggregates make processing and passaging difficult and introduce cell-cell contacts which likely lead to the contact-inhibition driven decrease in cell proliferation rate after day 4. Phase and fluorescent micrographs. Green $=$ LIVE $($ Calcein AM). Scale bar $=$ 
$1000 \mu \mathrm{m} .(k-n)$ cells were separated from microcarriers and stained with Hoechst 33342, and Nuclei were counted using

NIS Elements binary intensity thresholding. Scale bar $=100 \mu \mathrm{m}$. 


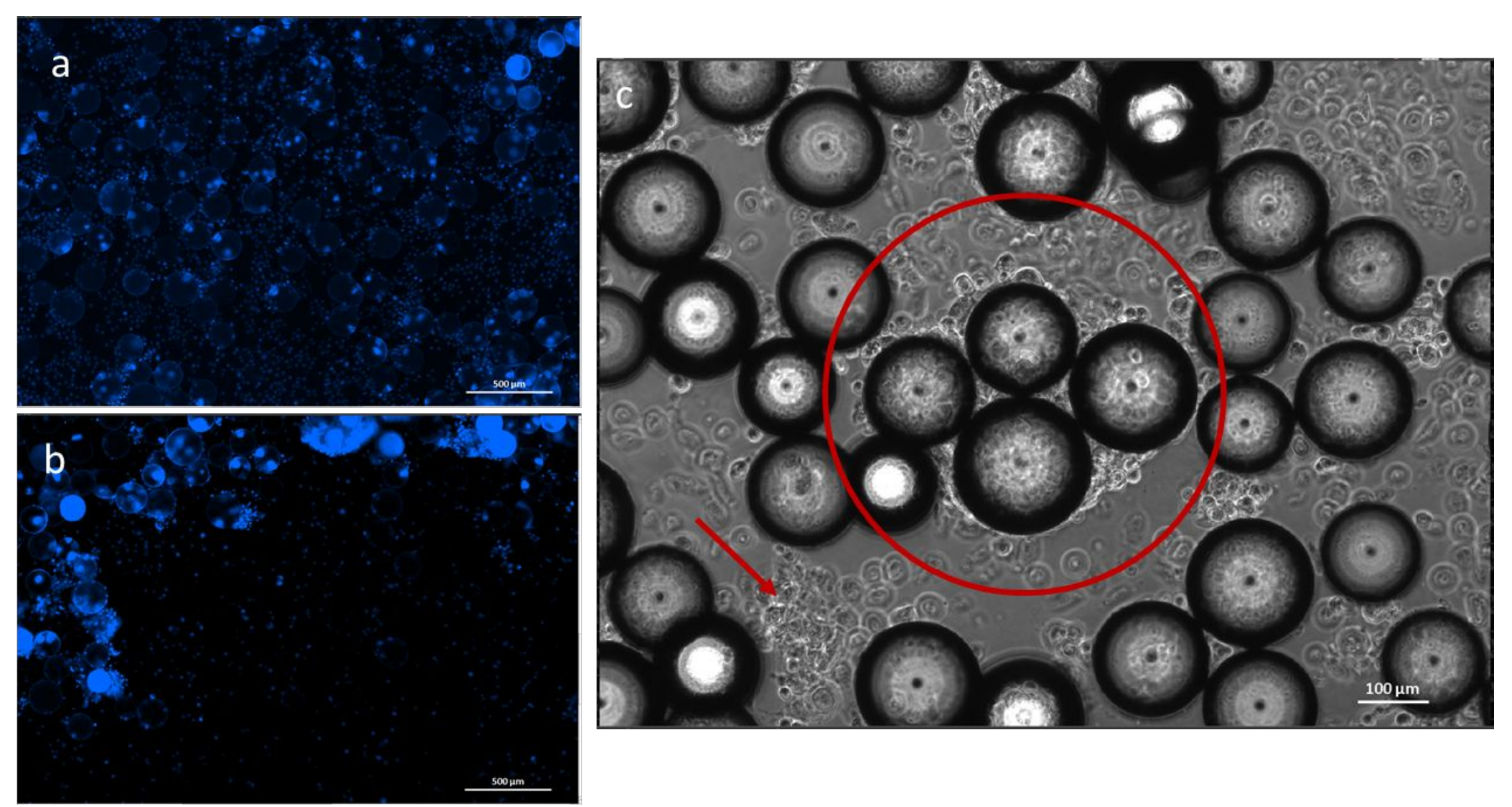

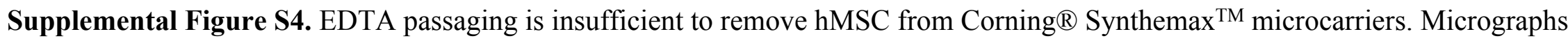
of hMSC grown on Synthemax ${ }^{\mathrm{TM}}$ microcarriers cultured in xeno free media, and passaged using a) TrypLE or b) EDTA. c) hMSC 
remain in large aggregates of cells alone (arrow) and cells and microspheres (circle). Blue $=$ DAPI, scale bars a,b) $1000 \mu \mathrm{m}, \mathrm{c}) 200$ $\mu \mathrm{m}$ 
A

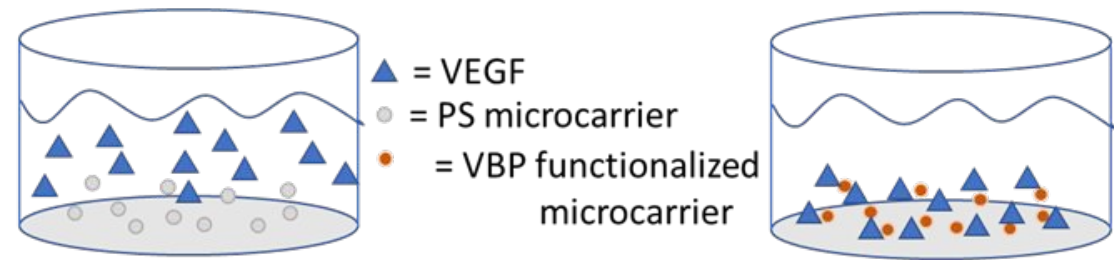

B

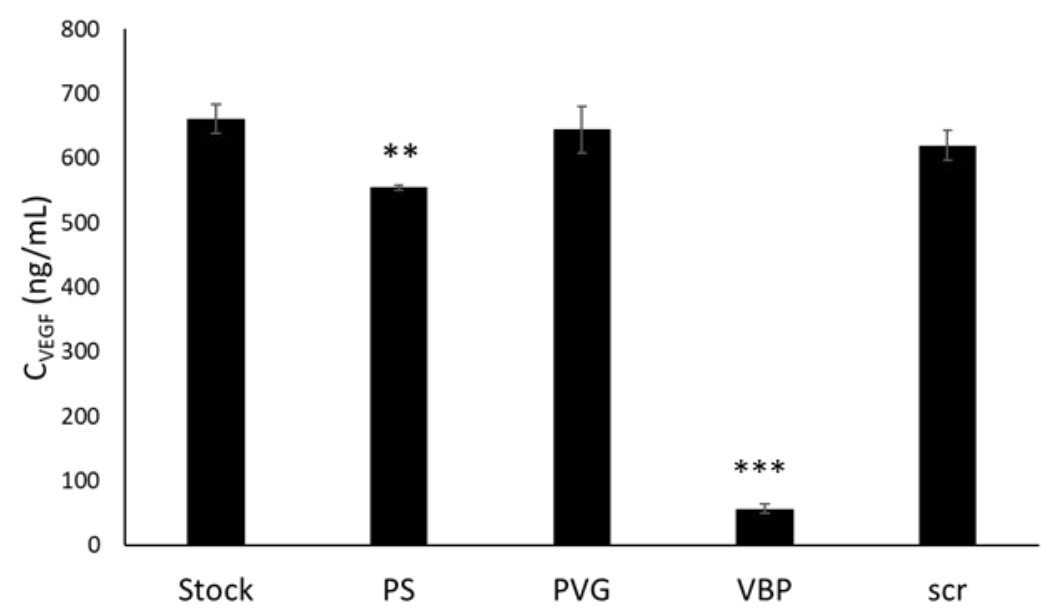

Supplemental Figure S5. VEGF Binding Peptide (VBP)-functionalized microcarriers can bind to soluble VEGF. a) VBP functionalized microcarriers bind to soluble VEGF, sequestering the protein near the culture surface. This results in b) a knockdown of soluble VEGF detectable by ELISA, where VBP functionalized microcarriers reduced significantly reduced the amount of detectable VEGF in the stock solution. ${ }^{*} \mathrm{p}<0.01 ; * * * \mathrm{p}<0.001$ compared to the stock solution by one-way ANOVA with post-hoc Tukey’s test. 